\title{
Knowledge Acquisition for Explainable, Multi-Expert, Knowledge-Based Design Systems
}

\author{
Rose Dieng, Alain Giboin, Paul-André Tourtier, Olivier Corby \\ SECOIA Project, INRIA - CERMICS \\ 2004 Route des Lucioles, 06561 VALBONNE CEDEX, FRANCE \\ E-mail: \{dieng, giboin, tourtier, corby\}@sophia.inria.fr \\ Fax: (33) 93657783
}

\begin{abstract}
In order to help the knowledge engineer and the expert during knowledge acquisition phase, the ACACIA Group is working on a knowledge acquisition methodology and tool (KATEMES) allowing knowledge acquisition from multiple experts, exploiting the specificities of design problems and preparing the assistance to the end-user and the quality of explanations he will be provided with. This paper describes our research program. After a brief description of our previous knowledge acquisition tool $S D K A T$, we will present the primitives of KATEMES and the problems we intend to study and the ideas we intend to deepen about the link between knowledge acquisition and explanations, knowledge acquisition from multiple experts and methodological aspects.
\end{abstract}

Keywords: knowledge acquisition and explanations, knowledge acquisition from multiple experts, knowledge graphs, cognitive agents, design applications

\section{Introduction}

During the phase of knowledge acquisition and specification of a multi-expert knowledge-based design system, how to prepare the quality of the final system (concerning as well its reasoning capabilities or the explanations it will be able to generate)? This question guides the research of ACACIA group, research summed up as follows:

In order to help the knowledge engineer and the expert during knowledge acquisition phase, we are working on a knowledge acquisition methodology and tool (KATEMES) allowing knowledge acquisition from multiple experts, exploiting the specificities of design problems and preparing the assistance to the end-user and the quality of explanations he will be provided with.

Some knowledge acquisition tools don't rely on the problem class while other researchers focus on a given problem class by proposing tools dedicated to this problem class: for example, $S A L T[50,51]$ is aimed at design applications. We will adopt this last approach and try to deepen design applications, for which our team 
had a number of experiments in the past $[59,58,60]$. Notice that very few knowledge acquisition tools concern design task, that is considered as very difficult to tackle: DSPL ACQUIRER [13], SALT [51], CANARD [69].

Here are the main questions we will study: 1) How to elicit and model knowledge from several experts? 2) How to take into account the users of the final knowledgebased system (KBS) and, in particular, to prepare the quality of the future explanations in the earlier knowledge acquisition phase? 3) How to validate the acquired knowledge? 4) How to design the final multi-experts system? 5) Can we propose a methodology taking into account explanations and multi-expertise?

KATEMES (Knowledge Acquisition Tool for Explainable, Multi-Expert Systems) will be the result of this research.

This paper will describe our research program. After a brief description of our previous knowledge acquisition tool $3 D K A T$, we will present the primitives of KATEMES and the problems we intend to study and the ideas we intend to deepen about the link between knowledge acquisition and explanations, knowledge acquisition from multiple experts and methodological aspects. Very-long-term research topics such as validation of the acquired knowledge and design of the final system will also be mentioned in order to give a complete idea of the ACACIA program. At the end of each section, we indicate related work, in order to recapitulate the points for which similar ideas were already evoked by other researchers and to highlight those for which we propose an original approach.

\section{From 3DKAT to KATEMES}

\subsection{DKAT}

In our past work, we developed a knowledge acquisition tool aimed at design applications, $3 D K A T^{1}[24,1]$. It allows to describe the structure of the designed system and of its components, and to represent explicitly the dynamic model used by the expert during the design process. It relies on the observation that, for a design application, the knowledge engineer seems to reconstruct a model of the system to be designed, using what he understood from the information provided by the expert. 3DKAT allows the knowledge engineer to make this model explicit and to let the expert validate it.

This model is based on the dependencies among the main parameters occurring during the problem solving and can be represented through a dependency graph called PDOG (Parameter Dependency Oriented Graph). A node of the graph corresponds either to an attribute of the object to be designed or to one of its components, or to a parameter issued from the external environment. 3DKAT proposes a typology of relations possible among the nodes. This hierarchy of links can be extended by application-specific relations. A particular relation, called topos, allows to express how a parameter influences another. The influence of a given parameter modification can then be visualized dynamically.

During knowledge acquisition phase, through a PDOG, the knowledge engineer

\footnotetext{
${ }^{1}$ 3DKAT was designed by Rose Dieng and Brigitte Trousse and implemented by Marie-Paule Epp, Nathalie Rjera and Eric Faisandier.
} 
makes the model he built from the interviews with the expert, explicit. Then he presents this dependency graph to the expert in order to complete it or correct it with him. Several types of qualitative reasoning are possible on the graph. The knowledge engineer can simulate the effect of increasing or decreasing a parameter (what-if reasoning), or simulate how to increase or decrease a parameter (how-to reasoning). Both kinds of reasoning are useful as well in design as in re-design from an existing solution. Thanks to a graphic, qualitative, parameterized simulation of the expert's reasoning, the macroscopic behaviour of the future expert system can thus be simulated and validated before effective implementation.

$3 D K A T$ also allows to model the subpart graph of an object as it is important in design applications. It offers a language of description of the expert's tasks and a notion of task graph, called TDOG (Task Dependency Oriented Graph).

The modelling of dynamic knowledge through PDOGs and the macroscopic validation thus allowed are one of the main interests of $3 D K A T$. Another strength is the ability to tackle some kinds of design tasks such as configuration of a composite system made of several components.

\subsection{Extensions of 3DKAT to KATEMES}

The part 2 of the SISYPHUS'91 project aimed at deepening various models of problem solving in knowledge-based systems, so as to analyse their influence on knowledge acquisition activities. A comparison of such models was performed through an example of office assignment. Our participation to this project [1] allowed us to analyse the differences between $3 D K A T$ approach and generic approaches such as Chandrasekaran's generic tasks [11,12] or KADS interpretation models [64]. We could also distinguish the aspects rather design-oriented in 3DKAT (the PDOGs, the subpart graphs) and the more general aspects (the task language, the use of semantic networks): PDOGs or subpart graphs are mainly useful in design problems consisting of building a composite system made of several components.

3DKAT allows to model one expert's vision only and the possibility of knowledge acquisition from several designers cooperating for a design task is not offered explicitly. Last, $3 D K A T$ does not take into account the future KBS user and it does not help the knowledge engineer to prepare the explanations this user would need.

Therefore, we will propose several extensions of $3 D K A T$ so as to constitute a new tool KATEMES (Knowledge Acquisition Tool for Explainable, Multi-Expert Systems). This new tool will be more ambitious than $3 D K A T$. It will also focus on design applications but in addition, it will tackle the problems of explanations and multi-expertise.

The following sections will describe the main planned research topics: a) choice of the primitives of the knowledge acquisition tool, b) preparation of the assistance to the end-user, c) study of multi-expertise, d) methodological aspects.

\section{The Primitives of KATEMES}

\subsection{Knowledge Graphs and Links}

KATEMES will offer the knowledge engineer a knowledge representation formalism 
and various libraries. Knowledge will be described through semantic networks, called knowledge graphs. Several types of knowledge graphs will be possible, according to the nature of nodes and links. The nodes of a knowledge graph can be: a) parameters of the system to be designed, b) tasks of the experts or of the users, c) agents (allowing to represent the experts).

As in MACAO [2], we will admit generic graphs (where nodes correspond to classes) and instantiated graphs (where nodes are associated to instances).

We will extend the taxonomy of links previously offered by 3DKAT: the knowledge engineer will then use this link library and will be able to complete it by application-specific links.

We will distinguish: a) static links (such as the subpart link): no behaviour will be associated to them and they will help to constitute static knowledge graphs. b) dynamic links (such as topoi): a behaviour will be associated to such links, so that a simulation can be performed on the dynamic graphs where such links will appear.

Some links will be general (interesting for various classes of problems) while others will be specially offered for design applications. Some links will be generic while others will be application-specific and added by the knowledge engineer.

Some kinds of important links will be stressed and considered as viewpoints: for example, electrical-link, mechanical-link, family-link are examples of such viewpoints that may be considered as interesting in an application. It will then be possible to associate knowledge graphs to these types of links. For example, in $3 D K A T$, there were three kinds of knowledge graphs: the subpart graph, the PDOG and the TDOG. The only kind of static link emphasized was the subpart link. The PDOG was gathering all kinds of links among parameters (allows-to-calculate, influence relation...). The TDOG was stressing links among tasks such as followed-by, ispalliated-by... In KATEMES, it will be possible to emphasize other kinds of links: instead of gathering all kinds of links in the PDOG, we will distinguish as many types of knowledge graphs as important links. For example, the knowledge engineer can decide to stress the links expressing "electrical relationships" and thus to handle an "electrical viewpoint knowledge graph".

Links can concern parameters or tasks or other entities. Links concerning entities of the same nature will lead to homogeneous knowledge graphs, while heterogeneous knowledge graphs will admit nodes of different natures.

As case-based reasoning is important in design applications [74,48], KATEMES will allow the knowledge engineer to elicit and store descriptions of typical solutions that may be used later for explanations. A typical solution will be a kind of knowledge graph, with a context describing its application conditions.

\section{Remark:}

We consider that this research, aiming at finding the adequate kinds of links and of knowledge graphs to emphasize, holds at a "knowledge level" [61]. We are aware of the complexity of this problem and we don't claim to find a general solution. But focusing on design applications should help us to determine links or knowledge graphs useful for this class of problems at least. 


\subsection{Tasks and Agents}

$K A T E M E S$ will offer a language for describing the experts' tasks (as in $3 D K A T$ ) and the users' tasks, and it will handle homogeneous knowledge graphs of tasks, as 3DKAT"s TDOGS.

To take into account multi-expertise, we will introduce the notion of agent, having knowledge graphs, representing its vision of the domain and of its reasoning in the domain. We will give a more detailed description of this notion of agent later, in the section concerning multi-expertise.

Some homogeneous knowledge graphs may have agents as nodes and represent networks of cooperating agents. Some heterogeneous graphs may emphasize the links among agents and tasks.

\subsection{Models of Reasoning}

$K A T E M E S$ will offer a qualitative reasoning on dynamic knowledge graphs. The relationships between the qualitative simulation offered by KATEMES and qualitative physics $[18,42]$ will be studied. In particular, we will compare our notion of knowledge graphs and the qualitative representation of a physical system.

A theoretical study of knowledge graphs, perhaps inspired of graph theory, will also be performed, as in [32].

We will study the consistency of KATEMES graphs through Petri nets: if we establish a correspondence between some classes of knowledge graphs and some known classes of Petri nets, we hope to take benefit of theoretical work already existing on Petri nets in order to deduce various properties of our knowledge graphs.

\subsection{Related Work}

The use of semantic networks or knowledge graphs for knowledge acquisition is quite frequent $[2,32]$ but proposing qualitative graphs on which a qualitative simulation is carried out seems an original idea, as well as the highlighting of particular links in a given knowledge graph. In [32], graph theory is used in order to analyse the properties of knowledge graphs.

Several typologies of links were already studied, in the framework of knowledge acquisition [2] or in a more general context [27,28].

\subsection{Conclusions}

We unify several notions in this basic notion of knowledge graph: the choice of the kinds of nodes, relations and knowledge graphs to emphasize for a given relation will result from a work at a knowledge level [61].

At the "symbol" level, we will use an object-oriented representation and implement agents, tasks, links, knowledge graphs as objects. 


\section{Knowledge Acquisition and Explanations}

There may be different types of end-users of the final KBS. Such users may have various purposes and need different forms of help. We rely on the principle that the quality of the final KBS future explanations must be prepared in the early knowledge acquisition phase: some information not necessarily useful for problem solving but important for the future explanations must be elicited from the experts. We will determine what this explanatory knowledge consists of and study how to elicit it, what influence it will have on the methodology and what formalisms will allow to represent it. This necessity to take into account the explanations as soon as the knowledge acquisition phase is recognized by researchers on explanations and the EES project $[57,56]$ relies on this idea.

\subsection{Explanatory Knowledge}

The analysis of the different steps leading to the production of an explanation (analysis of the user's request, generation of the explanation contents and presentation of the answer, using an adequate medium) can give indications on the explanatory information needed in each of such phases.

We will study the following points :

- Which explanatory knowledge elicit, when and how? In [14], the necessity to represent deep knowledge, domain principles, causal or mathematical models, and world knowledge for explanations was emphasized and is one of the interests of research on second generation expert systems [72]. So, deep models can be considered as explanatory information [14,15]. In [54], the author distinguishes missing knowledge (such as deep knowledge) and implicit knowledge embedded in the implementation (such as the rule choice criterion,...). In [57], different kinds of knowledge useful for explanations are stressed: domain model, domain principles, tradeoffs, preferences, terminological definitions, integration knowledge and optimization knowledge. In [20], the knowledge engineer must indicate application-specific relations, in prevision of future explanations. In [45], different types of explanatory knowledge useful for producing explanations are distinguished: explanatory principles, discourse structures, explanation strategies and factual knowledge.

Notice that explanatory knowledge may be dependent or not on the application or on the problem class: generic explanatory knowledge can thus be offered by a. knowledge acquisition tool while application-dependent explanatory knowledge needs be elicited from domain experts and from future users. We must study if this elicitation depends or not on the problem solving knowledge elicitation and if it must take place simultaneously or later. We must also study how to link explanatory knowledge to problem solving knowledge. If some explanatory information is stored in electronic documents, it is possible to link portions of text to the entities handled by the knowledge acquisition tool and to exploit hypertext links for later explanations.

- How to take into account the needs of the future user? Using elicitation techniques (interviews, activity analysis...) can allow to extract various information 
from the intended end-users of the final KBS: their expertise level in comparison with the system, the assistance they need from the system, their needs in explanations... We will study how the analysis of the end-users' activity can be integrated in the knowledge acquisition methodology. Determining the end-users' possible requests and the possible forms of answers they need will help the knowledge engineer specify the future user - KBS interaction and the system interfaces. He must also specify the way the system task will be integrated in the expert's one. The information elicited from the users may also be analysed to compare the users' knowledge and the experts' one about the domain and the problem solving, so as to exploit it in the explanatory module of the final KBS: for example, it could be a basis for correcting the misunderstandings of the non expert end-users of the final KBS or for filling in the deficiencies of such users. On the contrary, if the expertise level of the intended user is higher than the system's one, the system will be rather a kind of assistant for this user and offer him other kinds of explanations.

- How to elicit and combine the explanatory knowledge coming from several experts or users (consistency checking, choice between several explanations...)?

Remarks:

The cognitive study of explanatory dialogues among humans $[40,34]$ can give ideas on the adaptation of explanations to the interlocutor. The explanations given by the expert to another expert, to a non expert or to the knowledge engineer are examples of explanations offered to different types of users performing different tasks and having different goals when they ask explanations. The analysis of the differences of vocabulary and of explanatory strategies can indicate how the adaptation to the user is carried out. Some researchers consider the explanatory process as a cooperation among the explainer and the explainee: therefore, the nature and the role of both interlocutors affect the cooperation mode.

The interest of a multidisciplinary approach (in particular, the usefulness of cognitive psychology for knowledge acquisition) is more and more recognized [2,76].

\section{Conclusions}

Our study on explanatory knowledge should help us to propose:

- a typology of explanatory knowledge,

- a methodology taking into account the end-users,

- techniques of elicitation of explanatory knowledge from several experts,

- techniques for checking the consistency of explanatory knowledge elicited from several experts.

\subsection{Adaptation of Explanations}

We consider that explanations can depend on both the KBS task and the user's task. We will try to offer tools for improving the adaptation of the future explanations to the problem class or to the users. 


\subsubsection{Adaptation to Problem Classes}

Deepening classes of problems will allow us to constitute a library of "generic explanations". As a reflection on classes of problems and generic tasks was already carried out in the knowledge acquisition method $K A D S[9]$, we will rely on $K A D S$ generic tasks: our library will contain information on types of questions, users' goals, explanatory schemata and explanation presentation media advised for each $K A D S$ generic task.

For example, here is a list of various explanatory questions adapted to the design task [20]: a) the genesis and the evolution of designed objects [16], b) the relations between objects (cf their role, their evolution...), c) the different viewpoints on a given object, d) the comparison with typical solutions or typical cases, e) the justification of the choice between several alternatives, $f$ ) the failures, and in particular, those requiring a relaxation of constraints or a redesign, $g$ ) the transformation of a preliminary design into a detailed one, $h$ ) the influence of a modification of specifications or of constraints...

Discussions with some experts in civil engineering design convinced us that such experts often use analogies with typical solutions, when they give explanations to other experts of the same domain [16]. So, an explanatory mechanism for explaining some design choices may be based on such analogies, when the end-user is also an expert of the same domain.

As graphical explanations seem very useful for designers in civil engineering, graphics can be used as a medium of presentation, in addition to text.

Such examples of questions, explanatory mechanism and presentation medium for specific kinds of design task can be exploited for building the "generic explanations" associated to the design generic task.

Our library of "generic explanations" will contain such information, expressed using $K A D S$ vocabulary and it will guide the knowledge engineer for acquisition of explanatory knowledge, and for specification of an application-specific explanatory module.

\section{Remarks:}

This research of generic explanations associated to different classes of problems seems to be a new idea: generally, researchers study explanations regardless of the problem class. In [5], types of questions (such as "why metaclass" or "justify knowledge source" are associated to entities handled by $K A D S$, but without linking explanations to generic tasks of $K A D S$. The authors study model-based explanations, described at a knowledge level instead of a symbol level and we do approve this reflection at a knowledge level.

Most researchers don't take into account the problem class for explanations or study diagnostic tasks. Very few research on explanations tackle specificities of design task: let us cite $[38,21]$ and $[46]$ that is interested in the users of a CAD environment.

\subsubsection{Adaptation to the Users}

Many researchers study user models $[53,35,36,37,49]$. For a given application, some user's characteristics are interesting to be extracted and analysed during knowledge 
acquisition phase and stored. To deal with such users' characteristics, we will deepen the notion of user model [36] and try to offer a library of user models. We will distinguish generic user models and application-specific models and study techniques for acquiring and exploiting such user models.

In KATEMES, the viewpoint of a category of user will be constituted by its associated knowledge graphs. Techniques of comparison of knowledge graphs will allow us to compare the user's viewpoints and the expert's ones. We will then be able to determine a model common to the expert and to the user, as well as a model of the knowledge specific to each of them.

The correspondence between the users' viewpoints and the experts' ones may allow a better adaptation of the future explanations: for example, the KBS will be able to play on the vocabulary, the concepts understandable by a category of users, the simplified viewpoint that the user has on the expert's reasoning...

This study will end up in:

- a library of generic user models, that will be linked to the library of "generic explanations",

- techniques of comparison of users' knowledge graphs and experts' ones.

\subsection{Interest of KATEMES for Explanations}

The primitives of KATEMES are partly inspired from explanatory needs: for example, the description of typical solutions with their contexts of application is provided mainly for explanatory reasons, since references to typical cases seem to be often used by experts giving explanations to other experts in design applications [16].

The description of the tasks of the experts and of the end users can also facilitate the explanations, as well as the exploitation of different knowledge graphs extracted using KATEMES: simulations on knowledge graphs may sometimes be used as a kind of explanation.

During knowledge acquisition phase, we will allow the construction of a stractured documentation, based on hypertext techniques and useful later for the future explanations.

Among other modules, KATEMES will contain:

- a module allowing the exploitation of typical solutions for explanations,

- a module of exploitation of the knowledge graphs for explanations, with a specification of its use in the final KBS,

- a module exploiting such a hypertext-structured documentation, for explanations, with a specification of its use in the final KBS.

\subsection{Related Work}

The distinction between explanatory knowledge and problem solving knowledge was one of the basic ideas of the EES project $[57,56,73]$ and allows, in [62], to differentiate the explanation line from the reasoning line. 
For a second generation expert system, once acquired, the deep knowledge (i.e causal model or qualitative model of a system) will be available for later explanations. Model-based explanations are offered in [4].

In [52], explanations and knowledge acquisition are considered as two aspects of the same problem. Taking into account explanations in the earlier knowledge acquisition phase $[57,56,39]$ eases the later maintenance of the KBS. The notion of explanation structure extracted from the expert is proposed in [41]. In [30], the author proposes a knowledge acquisition technique based on elicitation of justifications from people. In [5], a model-based approach allows to anticipate the user's questions and to design mechanisms of answers as soon as the knowledge acquisition phase. Types of questions are identified and their semantics linked to the components of $K A D S$ method. Another way of linking knowledge acquisition and explanations is to exploit explanations in order to guide knowledge acquisition: $A D E L E$ [15] is a tool for helping to knowledge acquisition, relying on the exploitation of justifications or explanations in order to ratify knowledge. On the contrary, our approach consists of exploiting knowledge acquisition in order to prepare and improve future explanations.

The use of hypertext techniques for explanations was already proposed [33]. Such techniques are also more and more used for knowledge acquisition [43,44]: $K$-STATION (tool supporting the method KOD) and SHELLEY (tool supporting the method KADS) use such techniques. But the idea of exploiting the documents obtained during knowledge acquisition phase for later explanations don't seem to have yet been proposed.

\section{Knowledge Acquisition from Several Experts}

\subsection{The Problem}

The elicitation of knowledge from multiple experts was recently studied $[31,8,65$, $68,66,67,55,70,77]$. Sometimes, it is interesting to extract knowledge from several experts. They can be: a) either several experts working on the same domain and able to solve the same kinds of problems (but perhaps using different problem solving methods), b) or on the contrary specialists having different competence domains, each of them taking part in the solving of a more global problem.

The experts may disagree on the vocabulary or on some concepts or on limit cases. The knowledge engineer can try to model the common part of their knowledge as well as more specific aspects of this knowledge. He can detect possible conflicts and, according to the case, try to obtain a consensus or to keep the different viewpoints of the experts. So we must study how to represent the viewpoints of the different experts, the different possibilities of cooperation or of conflicts among the experts as well as the combination of their respective tasks.

We will study problems due to elicitation and analysis of knowledge of several experts: for example, can the analysis of the data elicited from an expert occur before and guide the elicitation of another expert knowledge or must the knowledge engineer avoid the biases that may be introduced by this way? We could benefit from previous experiments of knowledge elicitation from multiple experts [65] and from studies of cognitive psychologists for avoiding bias in knowledge elicitation. The 
analysis of the elicited data must allow to build: a) a common model corresponding to the kernel of knowledge common to all experts and perhaps models common only to sub-groups of experts, b) specific models corresponding to knowledge specific to an expert and not shared by other experts.

\subsection{Multi-Expertise in KATEMES}

The viewpoint of an expert will be constituted by his vision of the structural knowledge of the system to be designed (in the case of a design application), by his dynamic knowledge represented by dynamic knowledge graphs, by the description of his tasks and by his interactions with other experts.

We will compare the viewpoints of different experts, in order to detect and solve potential conflicts among them. Therefore, a correspondence may be established among the entities handled in the different knowledge graphs. We will propose techniques for managing consistency of several knowledge graphs.

We will extend the language of description of the expert's tasks, in order to take into account possible cooperation among several experts (for example, call to other experts, distribution of a task among several specialists...).

In order to tackle this multi-expertise problem, KATEMES handles the notion of agent to which a set of knowledge graphs will be associated. An agent may represent an expert totally or partially. More precisely, an expert may be represented by a compound agent, made of: a)an agent common to all experts, b) agents common to this expert and to other experts, c) and an agent representing the specificities of this expert.

The model of agent that we will propose will be described, among other features, by (a) its competences, (b) its vision of the domain and of its reasoning (cf its knowledge graphs), (c) its vision of other agents, (d) its interactions with the other agents [23].

In addition, we will propose a hierarchy of agents as well as a typology of relations that can link several agents. We will use this typology to describe semantic networks of agents [21,22], allowing to describe the way several experts work together, share their tasks, cooperate or communicate in real life.

Some knowledge graphs will have agents as nodes and represent networks of cooperating agents. We will study the possibility of qualitative simulation on agent knowledge graphs. The architecture of the final expert system will take inspiration of such networks if it intends to reflect the real behaviour of the experts in their activity.

\subsection{Notion of Composite System}

The cooperation of the users with the system can be described through a knowledge graph: the nodes will be agents representing the users or the system, and the arcs will be the relations between the users and the system.

In [23], we described our view of the process of knowledge acquisition as the behaviour of a composite system made of several human agents (experts, knowledge engineers and users) and software agents (the knowledge acquisition tool, the final system and the software where the final system will be integrated). This notion of 
composite system made of heterogeneous, interacting agents helps us to model the main relations between such agents (knowledge transfer, explanation, validation, assistance to problem solving...) and to analyse the cooperation underlying the process of knowledge acquisition. Our notion of agent should allow us to model the user as an agent and ease the description of a knowledge acquisition methodology involving several human agents.

\subsection{Related Work}

In the framework of distributed artificial intelligence $[7,29,19]$, we are related to research on distributed knowledge acquisition [26], that emphasizes the link between knowledge acquisition and multi-agent systems. The modelling of knowledge acquisition process using a composite system comprising interacting human or software agents is a new idea, as well as the notion of agent to which knowledge graphs are associated. The vision of the user as an agent of a composite system seems original.

A methodology for acquiring knowledge from a group of experts working together towards common goals is presented in [47]. The integration of knowledge from. multiple knowledge sources is studied in [55]. A method allowing to detect consensus, conflicts, correspondences et contrasts is proposed in [67]. Experiments based on grid repertories used by several experts are described in $[68,66]$. Techniques for comparing several viewpoints and solve conflicts among them are depicted in [25]. But techniques of comparison between several knowledge graphs representing the viewpoints of several experts don't seem to have been studied. The EMA knowledge acquisition method handles a notion of agent [71].

\subsection{Expected Results}

This research should lead us to propose:

- a formalism of multiple expert knowledge representation,

- a language of description of the tasks of several experts,

- a model of agent comprising, among others, a hierarchy of agents and a typology of relations among agents (cooperation, conflicts ...),

- techniques of comparison of several knowledge graphs,

- techniques for managing the consistency among the knowledge graphs.

\section{Methodological Aspects}

Several knowledge acquisition methods were proposed recently (EMA [71], KADS [9], KOD [76], MACAO [2]...) but none focus on design applications and takes into account the explanations and multi-expertise aspects. A top-down approach for knowledge acquisition for a design application was proposed in [10]. A few methodologies handle multiple experts explicitly $[68,47,77]$. EMA handles a notion of agent.

We will study methodological aspects, by taking into account the characteristics of design applications, explanations and multi-expertise. 
We already noticed several questions linked to the methodology: a) How to elicit and model knowledge stemming from several experts? b) When and how must the acquisition of explanatory knowledge be carried out? c) When and how must deep knowledge be elicited?

This seems rather ambitious. So, our work will be perhaps a study of the influence of such aspects on a methodology, and what extensions or deepenings the existing methodologies need to tackle such problems.

How would an original methodology be related to other existing methods? KADS proposes a general methodology, and adapts the knowledge acquisition mode to each identified generic task. So we will compare our approach with the propositions of $K A D S$ concerning design applications. $K O D$ is a general method for knowledge modelling and is independent of the kind of application. At present, we don't see any connection between our approach and $K O D$ approach. As $M A C A O$ is based on a cognitive model and aims at generality, we can study if this cognitive model needs to be adapted in order to take into account the specificities of design process.

\section{$7 \quad$ Very-Long-Term Research Topics}

\subsection{Validation of Acquired Knowledge}

The necessity to link knowledge acquisition and validation is recognized. During expertise transfer, the knowledge engineer will present the knowledge graphs obtained during the interviews, to the expert. In addition to this incremental validation of problem solving knowledge by the experts, explanations will have to be validated both by the experts and by the users.

Once the final KBS effectively built, the knowledge base will be validated relatively to the specifications constituted by the results of KATEMES. This link between knowledge acquisition, specification of the KBS and validation of the acquired and implemented knowledge is the basis of a "software engineering" approach during the development of the KBS.

We can also think about the a posteriori construction of the macroscopic description of an already existing expert system, developed without the help of KATEMES, in order to have it validated by the expert: it may be seen as a kind of "reverse engineering", allowing to verify if the KBS satisfies the macroscopic behaviour the expert had in mind, when describing his activity.

So we will propose rules of macroscopic validation of the KBS. In a very long term, we intend to exploit the research already performed in order to clarify the notion of validation and try to have a more formal vision of this notion $[3,63,6,75,17]$.

\subsection{Design of the Final Multi-Expert System}

Figure 1 describes our vision of the whole development of the final KBS.

The multi-expert aspect appearing in the knowledge acquisition phase can be translated in different architectures of the final KBS: several cooperating knowledge bases, a blackboard architecture, a multi-agent architecture... We don't intend to study these aspects. But in a very long term, we could study the possibility of automatic translation at least into the target shell SMECI that had been developed 
in our team [60]. Probably, this automatic translation will be able to tackle only a part of the final KBS.

Figure 2 sums up the main tools to be realized.

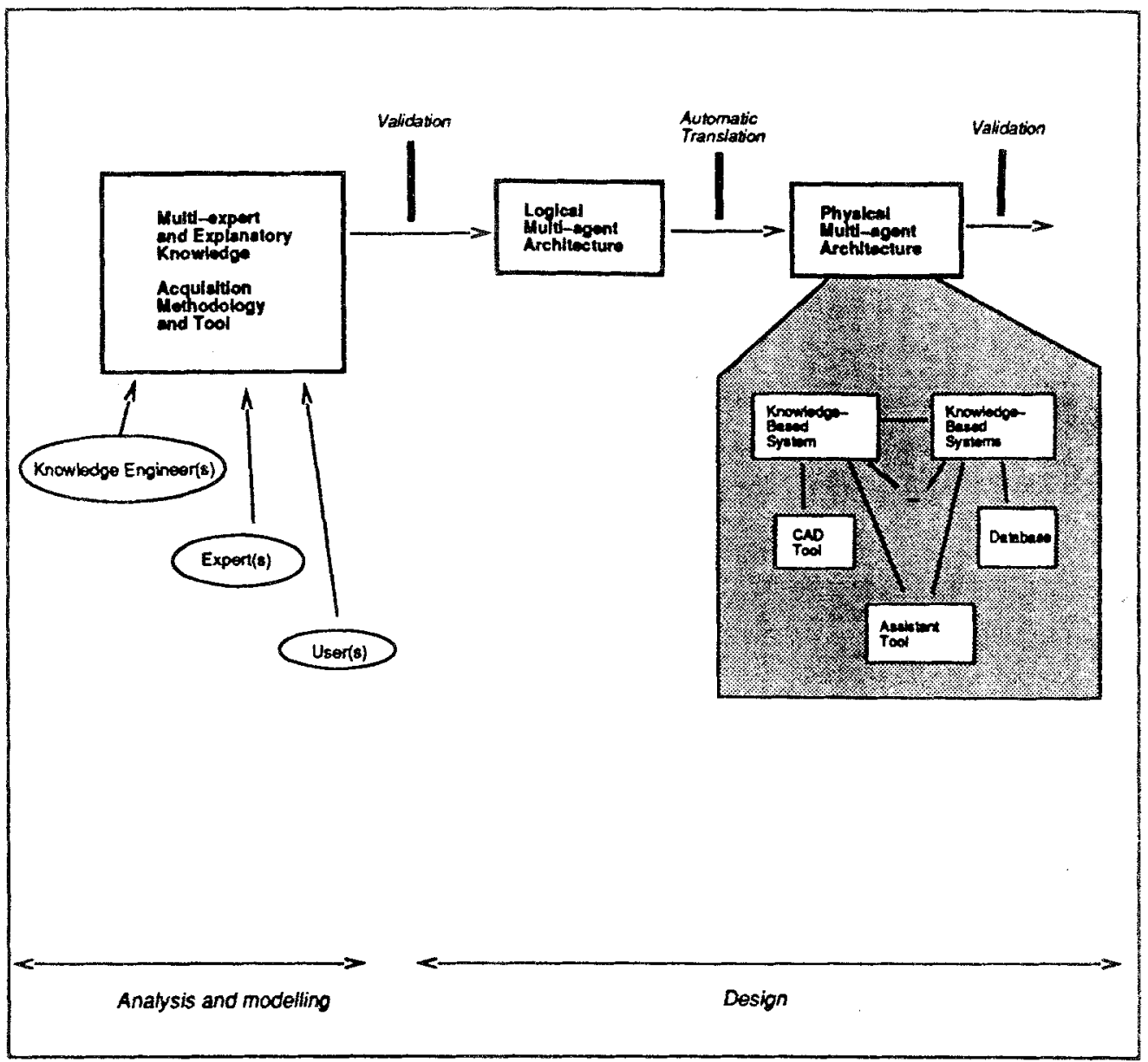

Figure 1: Phases of development of the multi-expert system 


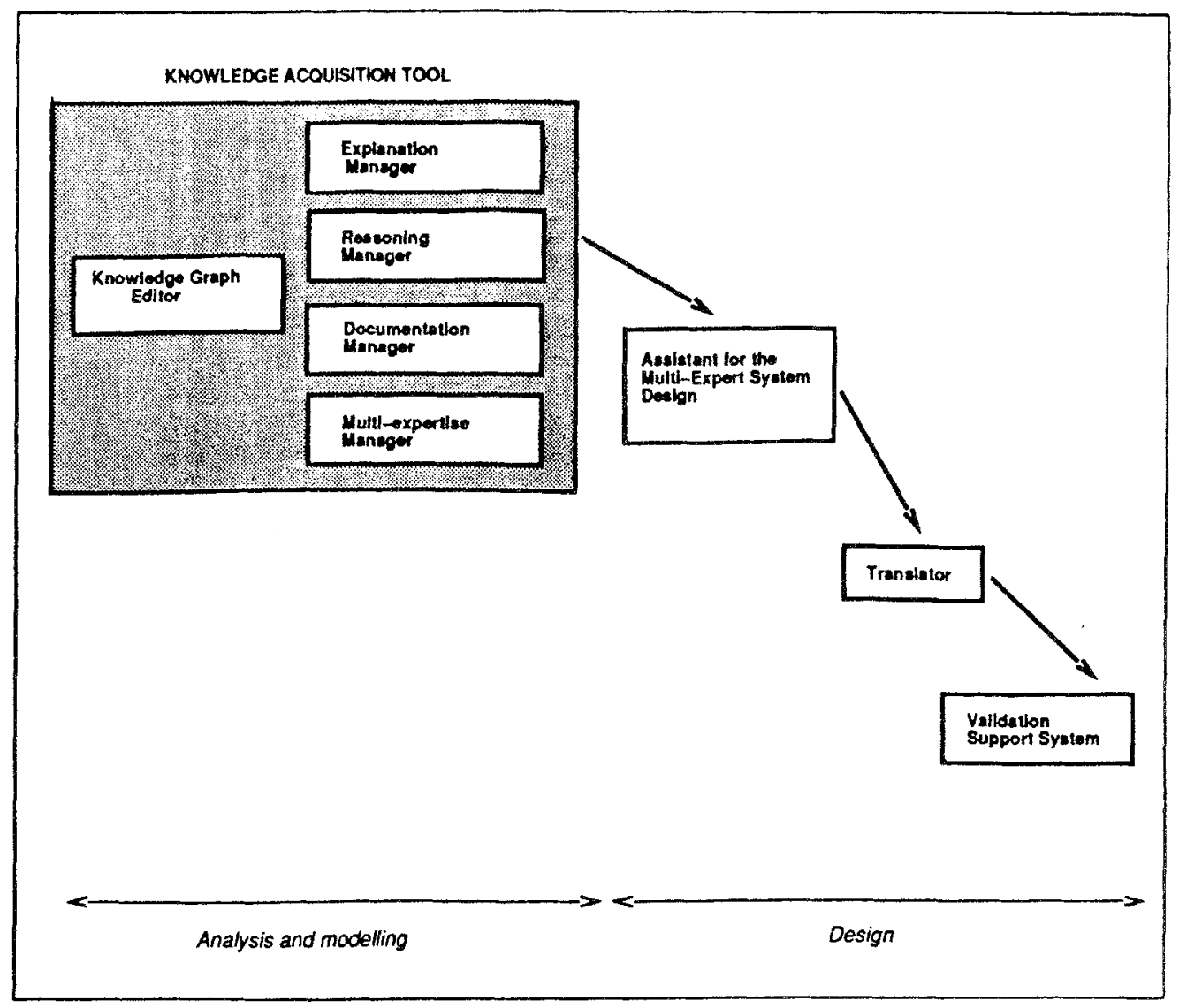

Figure 2: Tools to be realized

\section{Conclusions}

This paper presented our ideas on the various problems we intend to study in order to build KATEMES, a tool able to tackle specificities of design applications, explanations and multi-expertise. We are aware that this project is very ambitious and attacks several difficult problems. But it is important for us to think of such problems as soon as knowledge acquisition phase, so as to offer adequate primitives in our tool. Our previous experience in design applications, knowledge acquisition, explanations, and cooperation among cognitive agents will guide us. We will exploit, generalize and try to unify this previous work that inspired the key notions of KATEMES: agents, relations and knowledge graphs. The dependency on the problem class will help us to simplify some problems: each time we will try to build a library of generic, predefined entities (such as links, knowledge graphs, explanations...), we will deepen design applications, so as to offer the part of the library specially adapted to such applications, at least. 


\section{Acknowledgements}

We thank very much all our colleagues that helped us for the definition of this project:

- François Rechenmann for his careful rereading of the ACACIA program and his remarkable comments,

- Pierre Bernhard, André Bisseret, Gilles Kahn, Bernard Larrouturou and Alain Michard for their extremely judicious advices on the building of the ACACIA Group,

- the laboratories ARAMIIHS (in particular Nathalie Aussenac), LAFORIA (specially Patrick Brézillon) and LIRMM (in particular Danièle Hérin-Aimé, Joel Quinteton and Jean Sallantin) for fruitful contacts and discussions,

- Leila Alem, Franck Lebastard, Mireille and Claude Fornarino, Anne-Marie Pinna, Odile Pourtallier and the PhD students of the SECOIA team for their constant, friendly support,

- Christelle Amergé, Fatiha Belaid and Sofiane Labidi thanks to whom the ACACIA Group exists.

- Philippe Martin, Philippe Navarro and Jean-Christophe Pazzaglia who are implementing KATEMES.

\section{References}

[1] N. Aussenac and R. Dieng. Models of problem solving for knowledge acquisition: comparison of MACAO and 3DKAT. In SISYPHUS PROJECT, Part II, EKAW-91, Crieff, Scotland, UK, May 1991.

[2] N. Aussenac, J.L. Soubie, and J. Frontin. A mediating representation to assist knowledge with MACAO. In Proc. of $K A W \div 89$, pages 2.1-2.17, Banff, Canada, October 1989.

[3] M. Ayel. Protocols for Consistency Checking in Expert System Knowledge Bases. In Proc. of the 8th ECAI Conf., pages 220-225, Munich, August 1988.

[4] D.Y. Bau and P. Brézillon. Explanation-based diagnosis with the use of a model in control systems. In Proc. of the 11th Int. Workshop on Expert Systems and their Applications, Spec. Conf.: Artificial Intelligence \& Electrical Engineering, pages 3342, May 1991.

[5] A. Baumewerd-Ahlmann, P. Jaschek, J. Kalinski, and H. Lehmkuhl. Embedding Explanations into Model-Based Knowledge Engineering - Improved Decision Support in Environmental Impact Assessment. In D. Hérin-Aimé, R. Dieng, J.-P. Regourd, J.-P. Angoujard eds, Knowledge Modeling and Expertise Transfer, ISO Press, pages 269-284, Sophia-Antipolis, France, April 1991.

[6] I. Benbasat and J. S. Dhaliwal. The Validation of Knowledge Acquisition : Methodology and Techniques. In Proc, of EKAW-89, pages 60-74, Paris, July 1989.

[7] A. H. Bond and L. Gasser, editors. Readings in Distributed Artificial Intelligence. Morgan Kaufmann Publ., San Mateo, CA, 1988.

[8] J. H. Boose. A Survey of Knowledge Acquisition Techniques. Knowledge Acquisition, 1(1):3-37, March 1989.

[9] J. Breuker and B. Wielinga. Models of Expertise in Knowledge Acquisition. In Guida and Tasso, editors, Topics in Expert System Design. North-Holland, Elsevier Science Publishers B.V., 1989. 
[10] P. Buck, B. Clarke, and G. Lloyd. A Top-down Approach for Capturing Design Knowledge. In Proc. of EKAW-91, Crieff, Scotland, UK, May 1991.

[11] T. Bylander and B. Chandrasekaran. Generic Tasks in Knowledge-Based Reasoning : the 'Right' Level of Abstraction for Knowledge Acquisition. International Journal of Man-Machine Studies, 26:231-244, 1987.

[12] B. Chandrasekaran. Towards a Functional Architecture for Intelligence Based on Generic Information Processing Tasks. In Proc. of the 10th IJCAI, volume 2, pages 1183-1192, Milan, Italy, August 1987.

[13] T. Y. L. Chiang and D. C. Brown. DSPL ACQUIRER - A System of the Acquisition of Routine Design Knowledge. In Sriram and Adey, editors, Proc. of the 2nd Conference on Applications of Artificial Intelligence in Engineering, pages 95-110, Cambridge, MA, USA, August 1987. Mech. Comp. Publ.

[14] W. J. Clancey. The epistemology of a rule-based expert system - a framework for explanation. Artificial Intelligence, 20:215-251, 1983.

[15] M. O. Cordier and C. Reynaud. Knowledge Acquisition Techniques and SecondGeneration Expert Systems. In Proc. of EKAW-91, Crieff, Scotland, UK, May 1991.

[16] R. D. Coyne. Design Reasoning Without Explanations. AI Magazine, pages 72-80, Winter 1990.

[17] H. Davis. Using models of dynamic behavior in expert systems. In 9th Int. Workshop Expert Systems their Applications, pages 393-404, Avignon, France, May-June 1989.

[18] J. de Kleer and J. S. Brown. A qualitative physics based on confluences. Artificial Intelligence, $24: 7-83,1984$.

[19] Y. Demazeau and J.-P. Muller, editors. Decentralized AI, 2. North-Holland, Elsevier Science Publ., 1991.

[20] R. Dieng. Knowledge-based, relation-based and learning-based explanations. In Proc. of the 5th Workshop on Explanations, Manchester, UK, April 1990.

[21] R. Dieng. Relations linking Cooperating Agents. In Y. Demazeau and J.P. Muller, editors, Decentralized Artificial Intelligence, 2, Elsevier Science Publ. B.V., NorthHolland, 1991

[22] R. Dieng. Semantic network of expert systems. In Kohonen and Fogelman-Soulié, editors, COGNITIVA 90, North-Holland, Elsevier Science Publ. B.V., 1991

[23] R. Dieng and P.A. Tourtier. A Composite System for Knowledge Acquisition and User Assistance. In Proc. of AAAI Spring Symposium on Design of Composite Systems, pages 1-5, Stanford, CA, March 1991.

[24] R. Dieng and B. Trousse. 3DKAT, a Dependency-Driven Dynamic-Knowledge Acquisition Tool. In Proc. of the AAAI.88 Workshop on Integration of Knowledge Acquisition and Performance Systems, St-Paul, Minnesota, August 1988.

[25] S. Easterbrook. Handling conflict between domain descriptions with computersupported negotiation. Knowledge Acquisition, 3(3):255-289, September 1991.

[26] S. M. Easterbrook. Distributed Knowledge Acquisition as a Model for Requirements Elicitation. In Proc. of EKAW-89, pages 530-543, Paris, July 1989.

[27] M. Fornarino and A.M. Pinna. An original object-oriented approach for relation management. In Proc. of EPIA-89, Springer Verlag, Lecture Notes on Artificial Intelligence, n. 390, Lisbonne, Portugal, September 1989.

[28] M. Fornarino and A.M. Pinna. Un modèle objet logique et relationnel : le langage OTHELO. PhD thesis, Université de Nice, Avril 1990. 
[29] L. Gasser and M. N. Huhns, editors. Distributed Artificial Intelligence, vol. II. Pitman / Morgan Kaufmann Publishers Inc., London, 1989.

[30] T. Gruber. Justification-based Knowledge Acquisition. In H. Motoda, R. Mizoguchi, J. Boose, and B. Gaines, editors, Knowledge Acquisition for Knowledge-Based Systems (Proc. of JKAW-90), pages 148-158. IOS Press, 1991.

[31] V. Jagannathan and A. S. Elmaghraby. MEDKAT: multiple expert DELPHI-based Knowledge Acquisition Tool. In Proc. of the ACM NE Regional Conference, pages 103-110, Boston, MA, October 1985.

[32] P. James. Structuring Knowledge using Knowledge Graphs. In Proc. of EKAW-91, Crieff, Scotland, UK, May 1991.

[33] P. W. Jamieson. HyperExplain: A New Method for Natural Language Generation. In Proc. of the AAAI'88 Workshop on Explanation, pages 59-62, Saint-Paul, Minnesota, August 1988.

[34] H. Johnson and P. Johnson. Explanation Dialogues for Explanation and Learning (IDEAL): The Effects of Different Explanatory Dialogue Styles on Learning by NonExperts. In Proc. of the 5th Workshop on Explanations, Manchester, UK, April 1990.

[35] R. Kass and T. Finin. Rules for the Implicit Acquisition of Knowledge about the User. In Proc. of AAAI-87, volume 1, pages 295-391, Washington, DC, July 1987.

[36] R. Kass and T. Finin. Acquiring User Models for Tailoring Explanations. In Proc. of the AAAI'88 Workshop on Explanation, pages 48-54, Saint-Paul, Minnesota, August 1988.

[37] R. Kass and T. Finin. A general user modelling facility. In Proc. of the CHI'88, 1988.

[38] A. Kassatly and D.C. Brown. Explanation for Routine Design Problem Solving. In D. Sriram and R.A.Adey, editors, Knowledge Based Expert Systems in Engineering: Planning and Design, pages 225-239, Cambridge, MA, August 1987. Mech. Comp. Publ.

[39] C. Kellog, R. A. Gargan Jr, W. Mark, J. G. McGuire, M. Pontecorvo, J. L. Schlossbeg, and J. W. Sullivan. The Acquisition, Verification And Explanation Of Design Knowledge. SIGART Newsletter, Knowledge Acquisition Special Issue, (108):163-165, April 1989.

[40] A. Kidd. The Consultative Role of an Expert System. In P. Johnson and S. Cook, editors, People and Computers: Designing the Interface, Proc. of the Conference of the British Comupter Society, Human Computer Interaction Specialist Group, pages 249-254, University of East Anglia, September 1985. Cambridge University Press.

[41] O. Kuhn, M. Linster, and G. Schmidt. Clamping, COKAM, KADS and OMOS: The Construction and Operationalisation of a KADS Conceptual Model. In Proc. of EKAW-91, Crieff, Scotland, UK, May 1991.

[42] B. J. Kuipers. Qualitative simulation. Artificial Intelligence, 29:289-338, 1986.

[43] L. Lafferty, Jr Koller, A. M., G. Taylor, R. Schumann, and R. Evans. Techniques for capturing expert knowledge: an expert systems / hypertext approach. SPIE, Applications of Artificial Intelligence VIII, 1293:181-191, 1990.

[44] H. Langendø̈rfer, U. Schreiweis, and M. Hofmann. Knowledge Acquisition with a special Hypertext System. In H. Motoda, R. Mizoguchi, J. Boose, and B. Gaines, editors, Knowledge Acquisition for Knowledge-Based Systems (Proc. of JKAW-90), pages 249-258. IOS Press, 1991. 
[45] B. Lemaire and B. Safar. An architecture for representing explanatory reasoning. In D. Hérin-Aimé, R. Dieng, J.-P. Regourd, J.-P. Angoujard eds, Knowledge Modeling and Expertise Transfer, IOS Press, pages 327-332, Sophia-Antipolis, France, April 1991.

[46] S. Line and J. Ferrans. Explanation In a CAD Environment: Surveying User Requirements. In Proc. of the 5th Workshop on Explanations, Manchester, UK, April 1990.

[47] Y. I. Liou, E. S. Weber, and Jr J. F. Nunamaker. A methodology for knowledge acquisition in a group decision support system environment. Knowledge Acquisition, 2(2):129-144, June 1990.

[48] M.L. Maher. Process Models for Design Synthesis. AI Magazine, pages 49-58, Winter 1990.

[49] C. Maïs and A. Giboin. Helping users achieve satisficing goals. In M.J. Smith and G. Salvendy, editors, Work with Computers: Organizational, Management, Stress and Health Aspects, Advances in Human Factors/Ergonomics Series 12A (Proc. of the Third Int. Conf. on Human-Computer Interaction), pages 98-105, Boston, MA, September 1989. North-Holland, Elsevier Science Publishers B.V.

[50] S. Marcus. Taking backtracking with acquisition grain of SALT. International Journal of Man-machine Studies, 26(4):383-398, 1987.

[51] S. Marcus and J. McDermott. SALT: A knowledge acquisition language for proposeand-revise systems. Artificial Intelligence, 1988.

[52] B. Mark. Explanation and Interactive Knowledge Acquisition. In Proc. of the AAAI'88 Workshop on Explanation, Saint-Paul, Minnesota, August 1988.

[53] K. R. McKeown and R. A. Weida. Highlighting User Related Advice. In Proc. of the AAAI'88 Workshop on Explanation, pages 38-43, Saint-Paul, Minnesota, August 1988.

[54] C. Millet. A study of the knowledge required for explanations in expert systems. In Proc. of the 5th Conf. on Artificial Intelligence Applications, pages 83-90, Miami, Florida, March 1989.

[55] K. Morik. Integration Issues in Knowledge Acquisition Systems. SIGART Newsletter, Knowledge Acquisition Special Issue, (108):124-131, April 1989.

[56] R. Neches, W. R. Swartout, and J. Moore. Enhanced Maintenance and Explanation of Expert Systems through Explicit Models of their Development. IEEE Transactions on Software Engineering, SE-11(11):1337-1351, November 1985.

[57] R. Neches, W. R. Swartout, and J. Moore. Explainable (and maintainable) expert systems. In Proc. of the 9th IJCAI, pages 382-389, Los Angeles, CA, 1985.

[58] B. Neveu. EXPORT: an expert system in breakwater design. In ORIA 87, Artificial Intelligence and Sea, pages 11-17, 1987.

[59] B. Neveu and P. Haren. SMECI : an Expert System for Civil Engineering Design. In Proc. of the 1st Int. Conf. on Applications of Artificial Intelligence to Engineering Problems, Southampton, UK, April 1986.

[60] B. Neveu, B. Trousse, and O. Corby. SMECI : an Expert System Shell that fits Engineering Design. In 3er Simposium Internacional de Inteligencia Artificial, Monterrey, N.L., Mexique, October 1990.

[61] A. Newell. The knowledge level. Artificial Intelligence, pages 87-127, 1982. 
[62] C. L. Paris, M. R. Wick, and W. B. Thompson. The Line of Reasoning Versus the Line of Explanation. In Proc. of the AAAI'88 Workshop on Explanation, pages 4-7, Saint-Paul, Minnesota, August 1988.

[63] M. C. Rousset. On the Consistency of Knowledge Bases: the COVADIS System. In Proc. of the 8th ECAI Conf., Munich, 1988.

[64] G. Schreiber, B. Wielinga, and J. Breuker. The KADS Framework for Modelling Expertise. In Proc. of EKAW-91, Crieff, Scotland, UK, May 1991.

[65] M. L. G. Shaw. Problems of Validation in a Knowledge Acquisition System using Multiple Experts. In Proc. of EKAW-88, pages 5.1-5.15, Bonn, RFA, June 1988.

[66] M. L. G. Shaw. A Grid-Based Tool For Knowledge Acquisition : Validation with Multiple Experts. SIGART Newsletter, Knowledge Acquisition Special Issue, (108):168169, April 1989.

[67] M. L. G. Shaw and B. R. Gaines. A methodology for recognizing conflict, correspondence, consensus and contrast in a knowledge acquisition system. Knowledge Acquisition, 1(4):341-363, December 1989.

[68] M. L. G. Shaw and J. B. Woodward. Validation in a knowledge support system: construing consistency with multiple experts. International Journal of Man-Machine Studies, 29:329-350, 1988.

[69] D. B. Shema, J. M. Bradshaw, S. P. Covington, and J. H. Boose. Design knowledge capture and alternatives generation using possibility tables in Canard. Knowledge Acquisition, 2(4):279-390, December 1990.

[70] B. G. Silverman, R. G. Wenig, and T. Wu. COPEing With Ongoing Knowledge Acquisition From Collaborating Hierarchies of Experts. SIGART Newsletter, Knowledge Acquisition Special Issue, (108):170-171, April 1989.

[71] S. Spirgi and D. Wenger. Generic techniques in EMA: A model-based approach for Knowledge Acquisition. In Proc. of EKAW-91, Crieff, Scotland, UK, May 1991.

[72] L. Steels. Second Generation Expert Systems. In Future Generation Computer Systems, volume 1, pages 1213-1221, 1985.

[73] W. R. Swartout. Knowledge needed for expert system explanations. In AFIPS Conf. Proc., Nat. Computer Conf., pages 93-98, Chicago, IL, July 1985.

[74] K. Sycara and D. Navichandra. Integrating Case-Based Reasoning and Qualitative Reasoning in Design. In Computational Mechanics, editor, AI in Design, pages 231250, Southampton, UK, 1989.

[75] S. Twine. A Model for the Knowledge Analysis Process. In Proc. of EKAW-89, pages 253-268, Paris, July 1989.

[76] C. Vogel. How to Qualify Knowledge-Based Systems. In Proc. of the 4th COMPASS Conference (COMPASS-89), 1989.

[77] W. A. Wolf. Knowledge Acquisition From Multiple Experts. SIGART Newsletter, Knowledge Acquisition Special Issue, (108):138-140, April 1989. 